\title{
PHOTO-ELECTRIC PROPERTIES OF DIAMOND, MEASURED WITH A CRYSTAL COUNTER
}

\author{
by G. P. FREEMAN and H. A. VAN DER VELDEN \\ Physisch Laboratorium der Rijksuniversiteit te Utrecht, Nederland
}

\begin{abstract}
Synopsis
In this article measurements are described of the function of $\mathrm{H} \mathrm{e} \mathrm{c} \mathrm{h} \mathrm{t}$ for diamond. The result differs considerably from earlier measurements in which light or electrons are used to irradiate the crystal. Attention is drawn to the important fact that the photo-electric measurements in a crystal, performed with the aid of a crystal counter are more accurate and reliable than older methods.
\end{abstract}

1. Introduction. In the years $1920-1925 \mathrm{G} \mathrm{u} d \mathrm{~d}$ e $\mathrm{n}$ and $\mathrm{P} \mathrm{oh} \mathrm{l}$ and coworkers have done pioneer work with regard to the internal photo-electric phenomena in crystals ${ }^{1}$ ). A part of this work has found a theoretical climax in an article of $\mathrm{He} \mathrm{cht}{ }^{2}$ ) in which the relation is given between the current in a crystal, caused by a certain irradiation and the electric fieldstrength.

Suppose, $1^{\circ}$. that a voltage $V$ is applied over a crystal of thickness $d$ and that a number $n$ of electrons is raised to the conductionband at the cathode; $2^{c}$. that each of them will be able to move over a distance $l_{i}$ in the direction of the applied electric field. The contribution to the charge induced on the anode will be $Q=e \sum_{1}^{n} i l_{i} / d$. The distance $l_{i}$ generally depends on the fieldstrength in the crystal; this relation has been derived by $\mathrm{Hecht}$, taking into account certain conditions. In a recent paper by $\mathrm{McK}$ a $\mathrm{y}^{3}$ ) these conditions are amply put forward in connection with electron bombardment conductivity in diamond. Two of these conditions are of special importance:

1. The traps have to be evenly distributed throughout the whole crystal.

2. The effect of spacecharge as a result of trapped charges has to be negligible. 
When $v$ is the drift-velocity of the electron, $F$ the electric fieldstrength and $T$ the average life time of the electron in the conductionband, then the mean distance travelled by the electron in the direction of the electric field when the crystal is thick enough, is $\omega=v . F . T$. In addition we suppose that the positive charge does not contribute to the measured charge pulse. According to $\mathrm{He} \mathrm{cht}$ the mean distance $l$ travelled by the electron in the direction of the field is given by $l=\omega\left(1-e^{-d / \omega}\right)$. In our case we find a charge pulse $Q=n e \omega\left(1-e^{-d / \omega}\right) / d$ or $Q / n e=\Omega\left(1-e^{-1 / \Omega}\right)$ where $\Omega=v F T / d$.

By measuring $Q$ as a function of $F / d$ it is possible to find $v T$. That is what has been done in the experiments of $\mathrm{Gudden}$ and $\mathrm{Pohl}$, and $\mathrm{Mc} \mathrm{K}$ a y. Gudde $\mathrm{n}$ and $\mathrm{Poh} \mathbf{l}$ measured this relation for different crystals which were under a constant irradiation of ultraviolet light, so that they produced a continual electrical current. The formation of space charge was prevented by irradiation with red light during or between the experiments. In this way they tried to meet the second condition postulated. For diamonds this experiment ${ }^{4}$ ) gave a saturation field of $15 \mathrm{kV} / \mathrm{cm}$ and for $Q$ a half value of about $5 \mathrm{kV} / \mathrm{cm}$. M c K a y irradiated his crystals with electrons of 3 to $14 \mathrm{kV}$ and found about the same values as $\mathrm{Gudden}$ and $\mathrm{P}$ o h l. His method to diminish the influence of the space charge was much more complicated. He used an alternating voltage and a continuous irradiation with electrons in addition to it. Though this method has more degrees of freedom, at low values of the fieldstrength $\mathrm{M} \mathrm{c} \mathrm{K}$ a y's results are not in accordance with the results of $\mathrm{Gudde} \mathrm{n}$ and $\mathrm{Pohl}$. This can be reasonably explained by assuming that the compensation of the spacecharge has not been adequate enough.

We studied the same problem though we did not irradiate with ultraviolet light but with $\alpha$-particles. Our results showed a significant difference from those of former experiments. Our attention was drawn to this problem during an investigation ${ }^{5}$ ) concerning the removal of space charge in diamonds used as a crystalcounter, by red and infrared light. In these experiments we used a fieldstrength of less than $10 \mathrm{kV} / \mathrm{cm}$ and so it should be expected that an accumulation of spacecharge should give a regular diminishing of the magnitude of the charge pulses if the experiments were carried out in absence of red or infrared light. It came out however, that such a decrease was hardly observeable and only a rather rapid decrease in 
the number of chargepulses counted occurred as a result of this accumulation of spacecharge.

This decrease was rapid compared with the time during which the number of pulses remained practically constant and during this time the magnitude of the chargepulses remained as constant as the number. This is in contradiction with the results of $\mathrm{Gudden}$, $\mathrm{Pohl}$ and $\mathrm{McK}$ a y.

We determined the value of $v T$ again for diamond by measuring the mean maximum of the chargepulses for different values of the electric fieldstrength. According to our former investigation we removed the spacecharge by irradiation with red light. The diamond used was not the same one as in our other experiments.

2. The experimental arrangement. Our experiments were performed with the apparatus already described ${ }^{5}$ ), consisting of a slow linear ionisationchamber amplifier coupled to an oscillograph with photographical registration. This apparatus was very convenient for the determination of the distribution of the charge pulse magnitude, but we were mainly interested in the maximum magnitude of the chargepulses. For its determination we used a second apparatus consisting of an ionisation chamber amplifier with variable timeconstants in the differentiating and integrating circuits. This amplifier was coupled to a discriminator and a counter with which it was very easy to determine the maximum of the chargepulses. The time constant of our first apparatus was $10^{-3} \mathrm{sec}$. With the second amplifier we used a time constant of $10^{-5} \mathrm{sec}$. This gave a small decrease in the signal-to-noise ratio but had the big advantage of eliminating all troubles with accoustical and mechanical disturbances.

3. Results of the measurements. The measurements were done in two series namely: going from a low fieldstrength to a high one and vice versa. When the space charge is not sufficiently eliminated (e.g. by insufficient irradiation with red light) we must expect the result to depend on the way in which the crystal is used during preceding irradiation with $a$-particles. Consequently if this case existed there would be a difference between the two series of experiments. This was not the case.

The results are given in Fig. 1, adapted to the formula of $\mathrm{H} \mathrm{e} \mathrm{cht}$ From this Fig. 1 we can derive a value for $v T=5,3 \cdot 10^{-5} \mathrm{~cm}^{2} /$ Volt, taking into account that the thickness of the crystal is $0,1 \mathrm{~cm}$. $\mathrm{M} \mathrm{c} \mathrm{K}$ a y found an optimal value of $6,1 \cdot 10^{-6} \mathrm{~cm}^{2} /$ Volt. 
4. Discussion of our results. Qualitatively the curve of Fig. 1 is in good agreement with our previous result, that a rather sudden decrease in the number of pulses must be regarded as most characteristic for the accumulation of spacecharge during irradiation with $a$-particles. Indeed our results show that when using a fieldstrength of $5.000 \mathrm{~V} / \mathrm{cm}$, the internal field wil have to decrease to about 1.500 $\mathrm{V} / \mathrm{cm}$ before the signal will diminish rapidly.

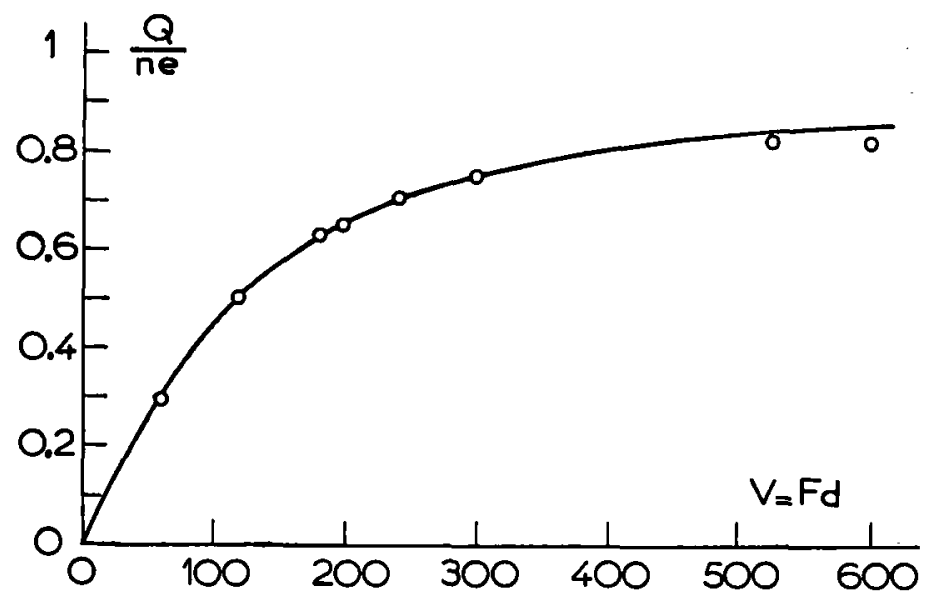

Fig. 1. Our measurements and the theoretical function of $\mathrm{Hecht}$.

Our experiments are in several respects different from those of $\mathrm{Gudde} \mathrm{n}$ and $\mathrm{Pohl}$ and of $\mathrm{McK}$ a y. They irradiate the total surface of the crystal and consequently average over this surface. We count separate $\alpha$-particles and in this way obtain a detailed image of a small fraction of the surface, e.g. by considering only the maximum deflection as in the curve mentioned. At the same time our method of removing the space charge differs from that of $\mathrm{M} \mathrm{c} \mathrm{K} \mathrm{a} \mathrm{y.} \mathrm{Our}$ method is not only simpler, but, according to our estimate, at least as good. The most essential difference is however, that we do not assume that all the electrons set free can travel through the whole crystal. This assumption was used by $\mathrm{Gudde} \mathrm{n}$ and $\mathrm{Poh} l$ as well as by $\mathrm{M} \mathrm{c} \mathrm{K} \mathrm{a} \mathrm{y} \mathrm{and} \mathrm{it} \mathrm{is} \mathrm{essential} \mathrm{to} \mathrm{a} \mathrm{supposed} \mathrm{even} \mathrm{distribu-}$ tion of traps. Our previous experiments about the influence of red light showed that, notwithstanding the fact that the $a$-particles of Po will all bring the same number of electrons to the conduction band, the resulting charge pulses are all of very different magnitude. 
Our method gives a good value for $v T$, assuming that the largest charge pulses consist of electrons that travel through the entire crystal. In other words: we assume that in the quantity $Q=e \sum_{i=1}^{n} l_{i} / d$ to be measured, only the values of $l_{i}$ are subject to large variations, whereas the number of electrons liberated is fairly constant.

For different reasons this must be considered very reasonable. It is known ${ }^{6}$ ) that our diamonds show a laminar structure; as a result of this structure internal cracks are very probable. Moreover diamond is a mixture of counting and not-counting parts providing for a higher trapping possibility, which then varies thoughout the volume of the diamond.

So we find that, according to our opinion at least, $\mathrm{M} \mathrm{c} \mathrm{K} \mathrm{a} \mathrm{y} \mathrm{and}$ $\mathrm{Gudden}$ and $\mathrm{P}$ oh 1 have measured a mean value of $v T$ which depends on the structure of the diamond. Our results may be interpreted to give at least a minimum value and probably the true value of $v T$. A further investigation is in progress to find out if this result is still dependent on structure or circumstances.

For large values of the electric field in the crystal there is a deviation of the magnitude of the signals from the formula of $\mathrm{H} \mathrm{e} \mathrm{ch} \mathrm{t}$. This can be easily understood by assuming that a disturbing surface exists parallel to the electrodes. A further indication bearing weight to this assumption may be found in the fact that in reversing the field on the crystal one finds much smaller signals.

The question may arise: what is the explanation of the small signals? Is it a question of reduction of mobility, or of a shorter time $T$ ? We are convinced that both facts are of importance. There are certain regions where internal cracks are preventing electrons to travel further in the conductionband. At larger fieldstrengths the distance travelled by the electrons will not increase; only the time $T$ will decrease. So the time $T$ is a function of $F$ and that is the reason why the theory of $\mathrm{H} \mathrm{e} \mathrm{c} \mathrm{h} \mathrm{t}$ is not applicable to the measurements hitherto made. Moreover in a region with more traps the mobility will be less than under the ideal conditions studied by us. This means that for larger values of the electric fieldstrength the number of large signals will increase since the electrons can travel further; this was proved experimentally to be true. At the same time one will never reach an ideal $G$ a u s s ian distribution because of internal cracks and similar deformities.

Taking a value for $V=156 \mathrm{~cm}^{2} / \mathrm{V} \mathrm{sec}$, as found theoretically by 
S e i t $\mathrm{z}^{7}$ ), the time $T$ during which the electrons are free to move throughout the conductionband will be $T=5,3 \cdot 10^{-5} / 156=3,5 \cdot 10^{-7}$ sec. The much larger value of $V=900 \mathrm{~cm}^{2} / \mathrm{V} \mathrm{sec}$, found according to $\mathrm{Seitz}{ }^{8}$ ) by $\mathrm{Klick}$ and $\mathrm{Maurer}$ in experiments concerning the $\mathrm{H}$ a 1 l-coëfficient gives the time $5,9 \cdot 10^{-8} \mathrm{sec}$.

It is evident from our experiments that one cannot speak of the mobility in diamond without further specification, namely: a mean value which is structure-dependent or an optimal value of the mobility. Both quantities are of interest depending on the kind of problem. One can evaluate the accuracy of our results for $v T$ to be about $20 \%$.

5. Conclusion and indication of further experiments. The method to investigate a crystalcounter with $\alpha$ - and $\beta$-particles gives a possibility for accurate measurements concerning the photo-electric properties of crystals. This is of importance both for the investigation of solids and for applications of the crystalcounter in nuclear physics. The distribution of the magnitude of the charge pulses found by van $\mathrm{Heerden}$ for $\mathrm{AgCl}^{9}$ ), Georgescu for $\mathrm{S}_{2}{ }^{10}$ ) (with $\beta$ particles) and $\mathrm{K}$ a $1 \mathrm{~m}$ a $\mathrm{n}$ for CdS (with $\alpha$-particles) makes it highly acceptable that one sometimes can use these crystals for energy measurements of radioactive radiation. Nevertheless it always seems to be necessary to investigate the homogenity and the number of traps in the crystal. Results by v a $\mathrm{n} \mathrm{H}$ e e r d e $\mathrm{n}$ show that with $\mathrm{AgCl}$ one can only expect reliable results with extremely good crystals. Because of a disturbing surface-effect it seems advisable to use $\beta$-radiation instead of $\alpha$-radiation.

For the measurement of photo-electric properties $\alpha$ - and $\beta$-radiation can be used and the results of this sort of measurements seem to be more reliable that with the usual photo-electric methods. This is the reason why we are extending our experiments to more diamonds and $\mathrm{AgCl}$ crystals and also that in addition we are using $\beta$-radiations.

In conclusion we wish to express our thanks and appreciation to Professor J. M. W. M i l a t z, for his continual and helpful interest throughout this work, and to the "Stichting Fundamenteel Onderzoek der Materie" that made this investigation possible under assistance from the „Nederlandse Organisatie voor Zuiver-Wetenschappelijk Onderzoek".

Received 19-5-50. 


\section{REFERENCES}

1) B. Gu d d e n und R. Poh l, Z. Phys. 16, 170, 1923.

2) K. He c h t, Z. Phys. 77, 232, 1932.

3) K. M c K a y, Phys. Rev. 74, 1606, 1948.

4) B. Gudde n und R. Poh l, Z. Phys. 17, 331, 1923.

5) H. A. v a n der Velden and G. P. Freem a n, Physica 16, 493, 1950.

6) C. V. R a m a n, Proc. Ind. Acad. Sci. Symposium I, 19A, 1944; Symposium II $24 \mathrm{~A}, 1946$.

7) F. Se it z, Phys. Rev, 73, 549, 1948.

8) F. Se it z, Phys. Rev. 76, 1376, 1949.

9) P. J. va n $\mathrm{H}$ e e $\mathrm{rde}$, The crystal counter, Thesis, Utrecht 1945.

10) Ge or ge s c u, C. R. Acad. Sci. Paris, 228, 383, 1949. 